\title{
The GNAQ in the haystack: intramedullary meningeal melanocytoma of intermediate grade at T9-10 in a 58-year-old woman
}

\author{
Michael Hoffmann, MD, ${ }^{1}$ Christian Koelsche, MD, ${ }^{2}$ Marcel Seiz-Rosenhagen, MD, ${ }^{3}$ \\ Sabine Mai, MD, ${ }^{1}$ Frank Lohr, MD, ${ }^{1}$ David Reuss, MD, ${ }^{2}$ Frederik Wenz, MD, ${ }^{1}$ \\ Christoffer Gebhardt, MD, ${ }^{4,5}$ and Frank A. Giordano, MD1
}

\begin{abstract}
Departments of ${ }^{1}$ Radiation Oncology, ${ }^{3}$ Neurosurgery, and ${ }^{4}$ Dermatology, Universitätsmedizin Mannheim, Medical Faculty Mannheim, Heidelberg University, Mannheim; 'Department of Neuropathology, Ruprecht-Karls-University Heidelberg, and Clinical Cooperation Unit Neuropathology, and DKTK, DKFZ, Heidelberg; and ${ }^{5}$ Skin Cancer Unit, German Cancer Research Center (DKFZ), Heidelberg, Germany
\end{abstract}

\begin{abstract}
Meningeal melanocytomas are rare tumors. They are derived from leptomeningeal melanocytes and predominantly occur along the spine and the posterior fossa. Here, the authors report a case of intramedullary melanocytoma of intermediate grade in a 58-year-old female patient who was initially misdiagnosed with malignant melanoma until mutational analyses of a panel of genes associated with melanotic tumors led to reclassification.
\end{abstract}

http://thejns.org/doi/abs/10.3171/2015.5.JNS15233

KEY WORDS meningeal melanocytoma; GNAQ mutation; CNS tumors; oncology

$\mathrm{M}$ ENINGEAL melanocytomas are extremely rare tumors originating from leptomeningeal melanocytes. The first reported description, in the early 1970s, was by Limas and Tio. ${ }^{10}$ Although little is known about this tumor entity as only a few case reports have been published over the past few decades, the tumors mostly occur along the spine and the posterior fossa with a peak incidence around the 5th decade and a slight female predominance. ${ }^{3,11}$

According to the classification suggested by Brat et al.,2 melanocytic neoplasms are categorized histologically as melanocytomas, melanocytomas of intermediate grade, or melanomas exhibiting typical malignant features such as necrosis and high mitotic activity. Recent in-depth molecular characterization of melanocytomas revealed distinct mutational and chromosomal fingerprints separating melanocytomas from other melanotic tumors such as melanoma metastases or melanotic schwannomas. ${ }^{6}$

Treatment aims at the complete surgical removal of the tumor and postoperative adjuvant radiotherapy.

\section{Case Report}

A 58-year-old woman presented with a history of progressive ataxia, progressive urinary and bowel inconti- nence, and weight loss of $6 \mathrm{~kg}$ over a period of $6-8$ weeks. Neurological examination revealed a paraparesis of the lower extremities (M0/5 muscle strength grade of the right leg; M1-2/5, left leg) with paresthesia and hypesthesia starting from the T-10 dermatome downward.

Her medical history included breast cancer diagnosed in 2008, which was treated with a lumpectomy, adjuvant chemotherapy (6 cycles of Taxotere, Adriamycin, and cyclophosphamide), and postoperative whole breast radiotherapy (including supraclavicular lymph nodes) with a total dose of 50.4 Gy and followed by a 16-Gy boost to the tumor bed.

Upon admission, MRI of the spine revealed a $3.7-\mathrm{cm}$ isodense/slightly hyperintense intramedullary lesion at T9-10. After administration of gadolinium, the lesion appeared hyperintense on T1-weighted images (Fig. 1A). T2-weighted images showed a myelopathy signal from T-7 to T-12. The differential diagnosis included ependymoma, astrocytoma, and intraspinal metastasis.

The patient thus underwent a laminectomy at the T9-10 levels and a complete microsurgical resection of the intramedullary tumor under continuous neuromonitoring. The lesion was grayish-red and heavily vascularized with a macroscopic appearance that closely resembled an epen- 

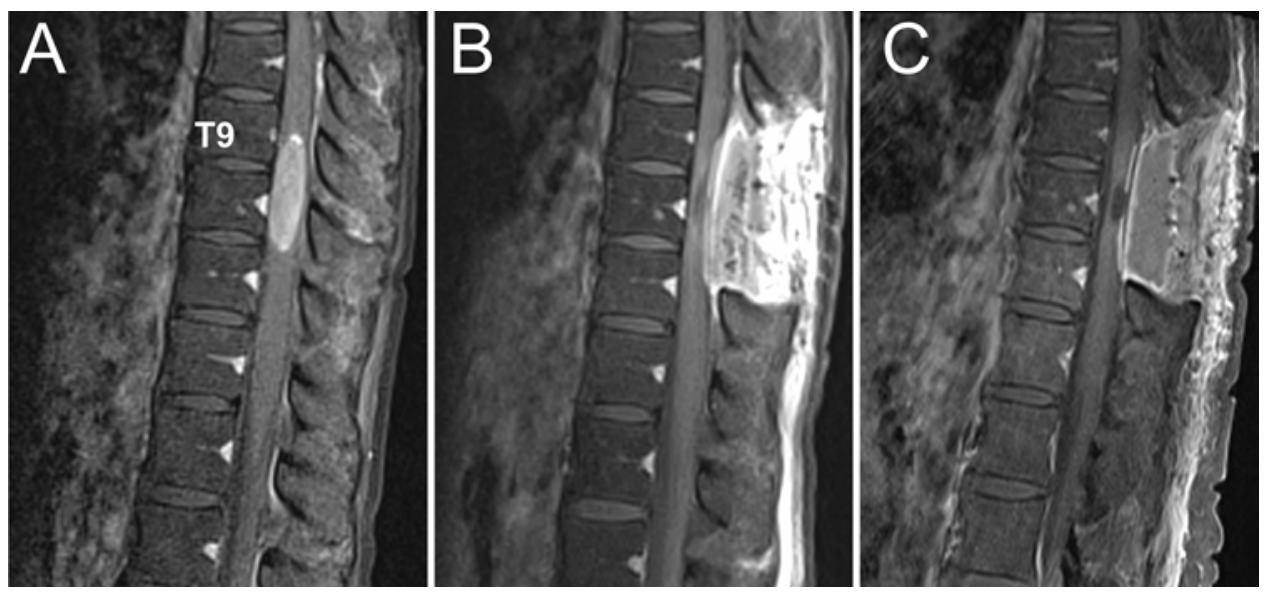

FIG. 1. Sagittal contrast-enhanced T1-weighted MR images of the thoracolumbar spine. A: Preoperative image showing the contrast-enhancing intramedullary tumor. B: Postoperative image showing the resection cavity and reactive contrast enhancement of the dorsal aspects of the spine. C: Image of the cavity at the first follow-up ( 3 months after surgery).

dymoma. A postoperative MRI scan showed no residual contrast-enhancing mass (Fig. 1B).

Histopathological workup showed a highly cellular tumor composed of epithelioid-shaped tumor cells with a scant to moderate cytoplasmic pigmentation and vesicular nuclei with prominent nucleoli (Fig. 2A). Single mitotic figures and small necrotic areas were present. The proliferation index Ki 67 (MIB1) focally reached 10\% (Fig. 2B). Tumor cells strongly expressed S100 and HMB45 (Fig. 2C and D), but were negative for BRAF V600E (VE1), pan-cytokeratin (AE1/3), EMA, and GFAP. Therefore, the tumor was classified as a melanoma of unknown primary (MUP).

However, neither dermatological and ophthalmological examinations nor imaging studies including CT from the neck to the pelvis and cerebral MRI revealed a primary tumor site. Based on the diagnosis of an MUP, the interdisciplinary tumor board recommended postoperative radiotherapy of T8-11 with 50 Gy in 25 fractions of 2.0 Gy followed by 4 cycles of ipilimumab.

One week prior to completion of radiotherapy, comprehensive molecular analyses of 6 key genes $(B R A F, N R A S$, cKIT, TERT promoter, GNA11, and GNAQ) revealed a hotspot missense mutation in codon 209 of the GNAQ gene (Fig. 3) while all other genes were not mutated (wild type). GNAQ mutations were previously found to be linked to uveal melanomas and melanocytomas, ${ }^{18}$ which can hardly be discriminated by histology alone. However, as ophthalmological consultation had already ruled out the presence of a uveal melanoma, the diagnosis of melanocytoma of intermediate grade was favored over MUP. Adjuvant treatment with ipilimumab was therefore omitted, and the radiation therapy was completed.

Radiotherapy was well tolerated by the patient, and the paraparesis improved only on the left side. At the end of radiotherapy, the patient could freely move her left leg (M3/5), while the right leg remained paralyzed (M0/5). The lower-limb paresthesia and hypesthesia, as well as the urinary and bowel incontinence, did not improve. At the first follow-up 3 months after the initial diagnosis, she presented with a persistent M3/5 of the left leg and a mildly increased strength grade (M1/5) of the right leg. Although the paresthesia had not improved, the patient regained bladder and bowel control. MRI of the total spine did not detect any remaining tumor (Fig. 1C) and a whole-body MRI scan showed no further lesions. After discharge, the patient was scheduled for quarterly follow-up visits including MRI of the total spine.

\section{Discussion}

Melanocytomas, especially of intermediate grade, and melanomas share a variety of histological features and therefore may be challenging for pathologists. Brat and colleagues $^{2}$ described low-grade melanocytomas as welldifferentiated lesions with tight nests, vasocentric fascicles, or sheet-like arrangements that typically lack malignant histological features such as high mitotic activity, necrosis, and nuclear pleomorphism (Fig. 2). Prominent nucleoli are a common feature of meningeal melanocytomas. ${ }^{16}$ Melanomas, however, consist of spindled or epithelioid cells with arrangements similar to those of melanocytomas, are densely cellular, and show significant cellular pleomorphism and nuclear atypia. Intermediate-grade melanocytomas, show histological features of aggressive behavior (necrosis, hypercellularity, mitosis, invasion of CNS structures) but lack the cellular atypia present in malignant melanomas. ${ }^{8}$

Immunohistochemically, both melanomas and meningeal melanocytomas are characterized by a positive staining for anti-melanoma antibody (HMB-45), S100 protein, and a negative reaction to EMA. ${ }^{16}$

Molecular analyses played a key role in establishing the more precise diagnosis for our patient. Without the detection of GNAQ mutation, our patient would have been misdiagnosed as having a malignant melanoma. In addition to uveal melanomas, blue nevi, and leptomeningeal or uveal melanocytomas, the GNAQ mutation may be found only in melanomas that developed from these benign precursors. ${ }^{6}$ Thus, in the absence of these lesions a GNAQ mutation is almost pathognomonic for melanocytoma. The GNAQ $(\mathrm{G}$ alpha q) gene is located on the long arm of chromosome 9 


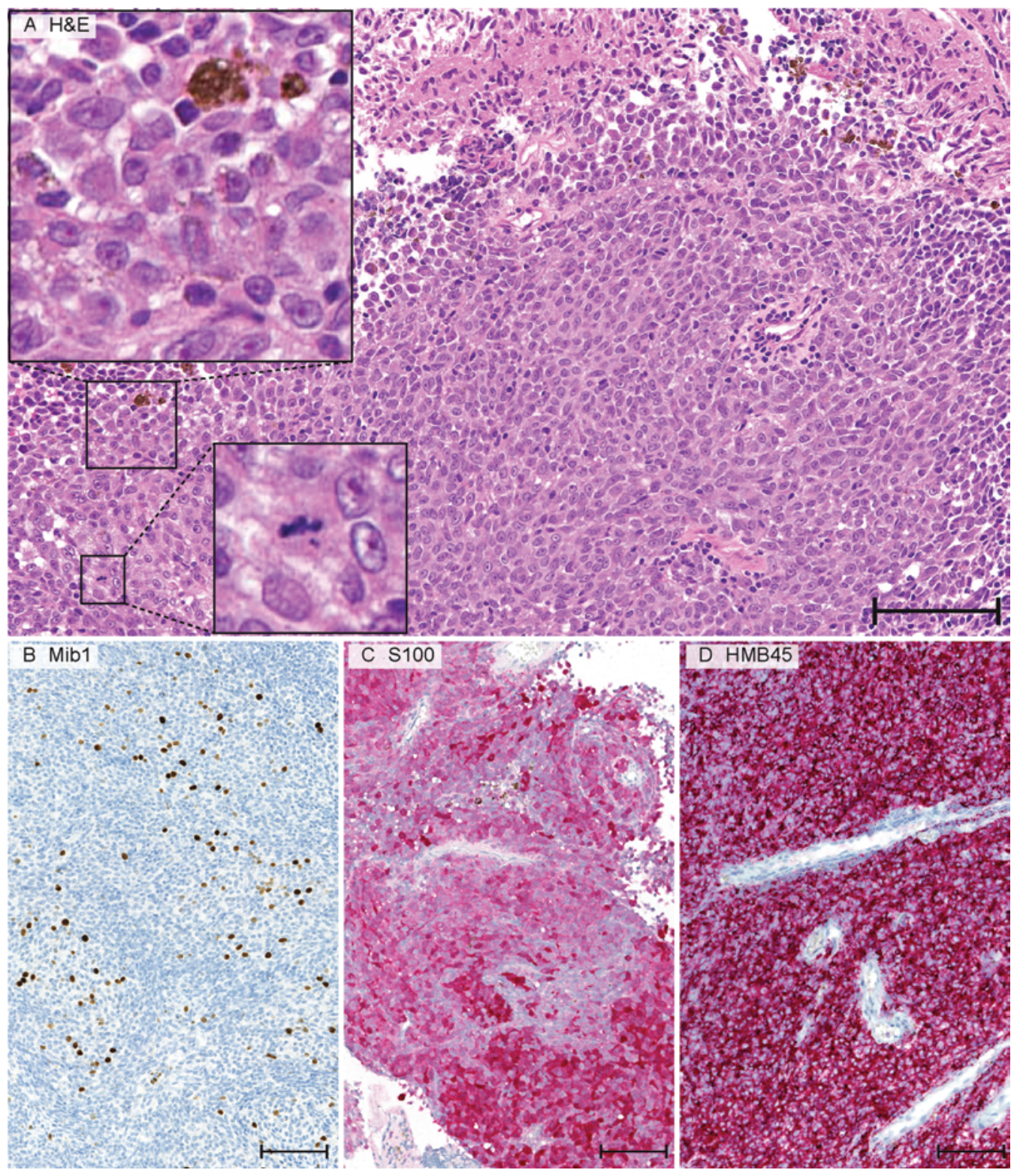

FIG. 2. Histological slides of the melanocytoma of intermediate grade. $\mathrm{H} \& \mathrm{E}$ stain showing a cellular, scant pigmented (upper inset) epithelioid tumor with single mitotic figures (lower inset) (A). The proliferation index Ki 67 (MIB1) was up to 10\% (B). Tumor cells were positive for S100 (C) and HMB45 (D). Original magnification $\times 200$; bar $=100 \mu \mathrm{m}$.

and encodes for a GTP-binding protein within the MAP kinase pathway. ${ }^{17}$ GNAQ codon 209 mutations (as detected in our patient) are found in pituitary and thyroid tumors, blue nevi, and ocular melanomas., ${ }^{912}$ The mutation results in constitutive activation of the protein, which permanently activates the MAP kinase pathway similar to $B R A F$ and NRAS mutations. ${ }^{14}$ Küsters-Vandevelde et al. ${ }^{7}$ suggested that the presence of a GNAQ mutation and the absence of typical mutations of cutaneous melanomas such as $B R A F$ or NRAS in a melanocytic neoplasm of the CNS strongly indicate a primary CNS tumor. On the other hand, the presence of a BRAF and NRAS mutation in the absence of a GNAQ mutation points to a primary cutaneous melanoma.

The symptoms of melanocytomas depend on the an- atomical site. The majority of patients will present with neurological symptoms caused by edema and compression of nerve bundles or brain tissue. In our case, spinal cord compression caused paraparesis, which partly resolved after combined treatment with surgery and radiotherapy. Different approaches to treatment were analyzed in a study by Rades et al. ${ }^{15}$ who showed that the 5-year survival rates were $100 \%$ for complete resection with or without adjuvant radiation. Incomplete resection with adjuvant radiation also achieved 100\% 5-year survival rates, whereas an incomplete resection alone only achieved $46 \%$ 5 -year survival rates. Therefore, the current gold standard of treatment for intracranial or intraspinal melanocytomas should be complete resection if possible. Due to 


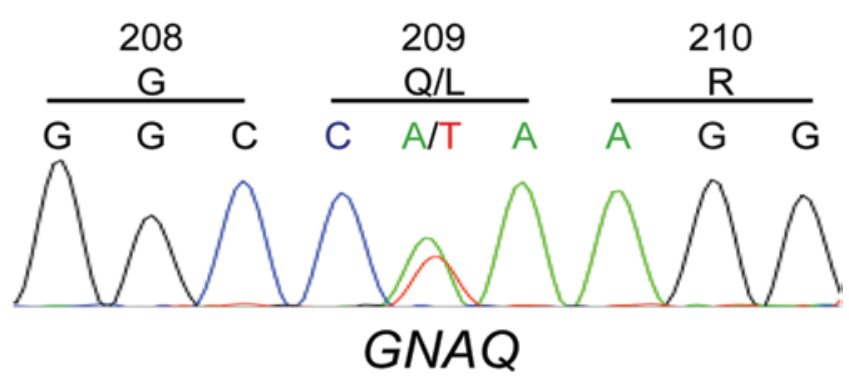

FIG. 3. Genetic analysis of the GNAQ gene. Sequencing of exon 5 of the GNAQ gene revealed a hotspot missense mutation in codon 209 (Q209 $L$ mutation).

the small number of cases, the role of adjuvant radiation therapy after complete resection is almost impossible to evaluate. Thus, in our opinion, radiotherapy should not be omitted for 2 reasons. First, although imaging techniques may have significantly improved and detection of residual tumor volume is more feasible nowadays, the definition of complete resection of a CNS tumor remains controversial in the absence of the possibility to assess a resection margin. Second, malignant transformation is possible,, 413 and metastasizing meningeal melanocytomas ${ }^{1,5}$ have been reported in the literature.

Our patient was treated with a radiotherapy regimen of 25 fractions of $2.0 \mathrm{~Gy}$, which was chosen at a time where the underlying tumor was believed to be a malignant melanoma. We have not observed radiotherapy-related adverse events so far, and, as Rades et al..$^{15}$ hypothesized an improved local control with doses exceeding $40 \mathrm{~Gy}$, we suggest that the "melanoma regimen" is a safe and practical approach for spinal meningeal melanocytoma.

Although this regimen appears to achieve high local control rates, we have initially scheduled the patient for quarterly follow-up visits as there is, in our opinion, not enough long-term data on these tumors available that would justify longer follow-up intervals.

In conclusion, we report on how molecular analyses helped establish the diagnosis of a meningeal melanocytoma of intermediate grade. In the absence of histologically confirmed (extra)cutaneous primary melanomas, we suggest a routine mutational analysis in the genetic loci of GNAQ (as well as BRAF, NRAS, $c K I T$, and GNAII) in all cases of melanocytic neoplasms of the CNS.

\section{References}

1. Barth A, Pizzolato GP, Berney J: [Intramedullary meningeal melanocytoma.] Neurochirurgie 39:188-194, 1993 (Fr)

2. Brat DJ, Giannini C, Scheithauer BW, Burger PC: Primary melanocytic neoplasms of the central nervous systems. Am J Surg Pathol 23:745-754, 1999

3. Brat DJ, Perry A: Melanocytic lesions, in Louis DN, Ohgaki $\mathrm{H}$, Wiestler OD, et al (eds): WHO Classification of Tumours of the Central Nervous System, ed 4. Lyon, France: IARC, 2007

4. Córdoba A, Tuñón T, Vázquez JJ: [Meningeal melanocytoma. Presentation of a case and review of the literature.] Arch Neurobiol (Madr) 52:93-99, 1989 (Span)

5. Delhaye M, Menei P, Rousselet MC, Diabira S, Mercier P: [A case of intramedullary primary melanocytic tumor: menin- geal melanocytoma or malignant melanoma?] Neurochirurgie 47:133-136, 2001 (Fr)

6. Koelsche C, Hovestadt V, Jones DT, Capper D, Sturm D, Sahm F, et al: Melanotic tumors of the nervous system are characterized by distinct mutational, chromosomal and epigenomic profiles. Brain Pathol 25:202-208, 2015

7. Küsters-Vandevelde HV, Klaasen A, Küsters B, Groenen PJ, van Engen-van Grunsven IA, van Dijk MR, et al: Activating mutations of the GNAQ gene: a frequent event in primary melanocytic neoplasms of the central nervous system. Acta Neuropathol 119:317-323, 2010

8. Kutzner H, Schärer L, Requena L: [Epithelioid and hyperpigmented melanocytic tumors. An overview.] Pathologe 28:411-421, 2007 (Ger)

9. Landis CA, Masters SB, Spada A, Pace AM, Bourne HR, Vallar L: GTPase inhibiting mutations activate the alpha chain of Gs and stimulate adenylyl cyclase in human pituitary tumours. Nature 340:692-696, 1989

10. Limas C, Tio FO: Meningeal melanocytoma ("melanotic meningioma"). Its melanocytic origin as revealed by electron microscopy. Cancer 30:1286-1294, 1972

11. Liubinas SV, Maartens N, Drummond KJ: Primary melanocytic neoplasms of the central nervous system. J Clin Neurosci 17:1227-1232, 2010

12. Lyons J, Landis CA, Harsh G, Vallar L, Grünewald K, Feichtinger $\mathrm{H}$, et al: Two $\mathrm{G}$ protein oncogenes in human endocrine tumors. Science 249:655-659, 1990

13. O’Brien TF, Moran M, Miller JH, Hensley SD: Meningeal melanocytoma. An uncommon diagnostic pitfall in surgical neuropathology. Arch Pathol Lab Med 119:542-546, 1995

14. Pollock PM, Harper UL, Hansen KS, Yudt LM, Stark M, Robbins CM, et al: High frequency of BRAF mutations in nevi. Nat Genet 33:19-20, 2003

15. Rades D, Schild SE, Tatagiba M, Molina HA, Alberti W: Therapy of meningeal melanocytomas. Cancer 100:24422447, 2004

16. Rahimi-Movaghar V, Karimi M: Meningeal melanocytoma of the brain and oculodermal melanocytosis (nevus of Ota): case report and literature review. Surg Neurol 59:200-210, 2003

17. Ross EM, Wilkie TM: GTPase-activating proteins for heterotrimeric $\mathrm{G}$ proteins: regulators of $\mathrm{G}$ protein signaling (RGS) and RGS-like proteins. Annu Rev Biochem 69:795-827, 2000

18. Van Raamsdonk CD, Bezrookove V, Green G, Bauer J, Gaugler L, O'Brien JM, et al: Frequent somatic mutations of GNAQ in uveal melanoma and blue naevi. Nature 457:599602,2009

\section{Disclosure}

Dr. Lohr reports receiving non-study-related support from Elekta and IBA, owning stock in Medigene and IMuc, and being on the board of C-Rad.

\section{Author Contributions}

Acquisition of data: Koelsche. Analysis and interpretation of data: Giordano, Hoffmann, Koelsche, Lohr, Gebhardt. Drafting the article: Giordano, Hoffmann, Gebhardt. Critically revising the article: Giordano, Koelsche, Seiz-Rosenhagen, Lohr, Reuss, Wenz, Gebhardt. Reviewed submitted version of manuscript: all authors. Approved the final version of the manuscript on behalf of all authors: Giordano. Administrative/technical/material support: SeizRosenhagen, Mai, Reuss, Wenz. Study supervision: Giordano.

\section{Correspondence}

Frank A. Giordano, Department of Radiation Oncology, Universitätsmedizin Mannheim, Medical Faculty Mannheim, Heidelberg University, Theodor-Kutzer-Ufer 1-3, Mannheim 68167, Germany.email: frank.giordano@umm.de. 